\title{
A lifetime measure for telecommunication network: Theoretical aspects
}

\author{
Zari Dzalilov, Iradj Ouveysi and Alexander Rubinov, \\ Centre for Informatics and Applied Optimization, \\ School of Information Technology and Mathematical Sciences, \\ University of Ballarat, Victoria, Australia
}

\begin{abstract}
A new measure for network performance evaluation called topology lifetime was introduced in [4], see also [5]. This measure is based on the notion of unexpected trafe growth and can be used for comparison of topologies. We discuss some advantages and disadvantages of the approach from [4] and suggest some modications to this approach. In particular we discuss how to evaluate the in uence of a subgraph to the lifetime measure and introduce the notion of the order of a path. This notion is useful if we consider a possible extension to the set of working paths in order to support the trafc for the time that is needed for installation of new facilities.
\end{abstract}

\section{INTRODUCTION}

The ability of a telecommunication network to support the expected growth in demand is an important characteristic of the network (see, for example, [1], [2], [3]). However, it is not enough to consider only the expected growth: an unexpected growth can often occur due to some technological innovations and the increasing popularity of the internet (see [4] for a corresponding discussion). A quantitative measure for telecommunications topology design was suggested by $\mathrm{N}$. Maxemchuk, I. Ouveysi and M. Zukerman in [4]. This measure was called topology lifetime. Possible unexpected changes in load have been taken into account in [4]. Note that the topology lifetime measure depends not only on topology but also on the set of working paths. In the current paper we discuss some advantages and disadvantages of the approach from [4] and its possible modi cations. Two other topics are also discussed in this paper. The rst is the notion of the order of a path (a special measure for comparison of paths). We need such a measure for the evaluation of paths that can be added to the set of working paths, if it is necessary to support the traf $c$ when large unexpected changes in load arise. The second topic is the evaluation of the lifetime measure of a sub-network of the given network. We use some modi cation of the approaches from [6] for this purpose. We concentrate mainly on theoretical aspects of the problem. The topic of further research is to apply the proposed approaches to some real world topologies and analyze the results obtained.

\section{Preliminaries}

Consider a network de ned by the graph $G=\langle V, E\rangle$ with the set $V$ of vertices (nodes) and the set $E$ of edges (arcs, links). Our approach is suitable for both directed and undirected topologies. For the sake of de niteness we will consider undirected topologies, so we consider $G$ as a nonoriented graph. Assume that capacities $c_{k, l}((k, l) \in E)$ are given. Since we consider an undirected topology, it follows that $c_{k, l}=c_{l, k}$. Recall that a path $p=\left(v, i, \ldots, j, v^{\prime}\right)$ between $v, v^{\prime} \in V$ is a sequence of links $(v, i),(i, k), \ldots,\left(j, v^{\prime}\right)$, which does not contain cycles. The number of links in $p$ is called the length of $p$. Since the graph $G$ is non-oriented, a path $\left(v, i, \ldots, j, v^{\prime}\right)$ coincides with the path $\left(v^{\prime}, j, \ldots, i, v\right)$.

The set of all paths generated by $G$ is denoted by $P(G)$. We take into account only a small subset $P^{*}(G)$ of working paths. We use the following notation:

$P_{k, l}^{*}(G)$ is the set of paths from $P^{*}(G)$, which contain the link $(k, l)$;

$P^{*}(i, j ; G)$ is the set of paths from $P^{*}(G)$ with the endpoints $i$ and $j$.

Denote by $x_{p}$ the amount of ow sent through a working path $p$. The totality $\left(x_{p}\right)_{p \in P^{*}(G)}$ of all ows is called a traf c generated by set of working paths $P^{*}(G)$. We will consider only feasible traf cs $\left(x_{p}\right)_{p \in P^{*}(G)}$. Feasibility means that $x_{p} \geq$ 0 for all $p \in P^{*}(G)$ and

1) the total ow transmitted through each link $(k, l) \in E$ does not exceed the capacity $c_{k, l}$ of this link:

$$
\sum_{p \in P_{k, l}^{*}(G)} x_{p} \leq c_{k, l}, \quad(k, l) \in E .
$$

2) the traf $c$ requirement constraints are satis ed.

In order to explain 2) we assume that the present traf c demand is represented by a nite collection $\mathcal{T}$ of traf $\mathrm{c}$ matrices $T$. Each $T \in \mathcal{T}$ describes the traf $\mathrm{c}$ demand between all OD pairs at a certain time period. Let $T=\left(t_{i j}\right)$. Then $t_{i j}$ is the traf $\mathrm{c}$ demand from node $i$ to node $j$ at the time period under consideration. Since we consider undirected traf $c$, we have $t_{i j}=t_{j i}$. Clearly $t_{i i}=0$ for all $i$. Traf c $\left(x_{p}\right)_{p \in P^{*}(G)}$ satis es traf $c$ requirement constraints if

$$
\sum_{p \in P^{*}(i, j ; G)} x_{p} \geq t_{i, j}
$$

for all $i, j \in V, i \neq j$ and for all $T=\left(t_{i j}\right) \in \mathcal{T}$.

\section{FEASIBILITY AND GROWTH FACTOR OF TRAFFIC MATRIX}

Consider a traf c matrix $T \in \mathcal{T}: T=\left(t_{i j}\right)$. Let $P^{*}(G)$ be the set of working paths. Consider the system of linear 
inequalities:

$$
\begin{gathered}
\sum_{p \in P^{*}(i, j ; G)} x_{p} \geq t_{i, j}, \quad i, j \in V, i \neq j \\
\sum_{p \in P_{k, l}^{*}(G)} x_{p} \leq c_{k, l}, \quad(k, l) \in E \\
x_{p} \geq 0, \quad p \in P^{*}(G) .
\end{gathered}
$$

If this system has a solution, the matrix $T$ is called a feasible matrix. (Sometimes the different de nition of feasibility is used, where the inequality in (1) is replaced with the equality. However the form of feasibility which we use is more natural.)

The growth factor $\psi_{*}(T)$ of the traf c matrix $T$ ( see [4]), is the the largest number $\psi$ such that the traf c $\psi T=\left(\psi t_{i, j}\right)$ is feasible. (The authors of [4] used the de nition of feasibility with equalities in (1).) Thus $\psi_{*}(T)$ is a solution of the linear programming problem:

$$
\text { maximize } \psi \text { subject to }\left(\left(x_{p}\right)_{p \in P^{*}}, \psi\right) \in X(T)
$$

where $X(T)$ consists of all the collections $\left(\left(x_{p}\right)_{p \in P^{*}}, \psi\right)$ such that $\psi \geq 0$, (2) holds and

$$
\sum_{p \in P^{*}(i, j)} x_{p}=\psi t_{i, j}, \quad i, j \in V, i \neq j
$$

The number $\psi_{*}(T)$ indicates the biggest possible uniform growth of the traf c $T$. The number $\psi^{*}(\mathcal{T})=\min \left\{\psi^{*}(T)\right.$ : $T \in \mathcal{T}\}$ is the biggest possible uniform growth of the traf $\mathrm{c}$ represented by the collection $\mathcal{T}$.

The de nition of the growth factor is based on a multiplicative approach to traf $c$ extension, since we consider the products of the form $\psi \cdot t_{i, j}$. One of possible approaches to the lifetime measure is to consider an optimization problem with constraints (3) where not only $x_{p}$ and $\psi$ but also $t_{i j}$ are variable. Such a problem arises if we want to estimate the worst performance with the respect to a set of matrices $T$. Unfortunately constraints (3) are bilinear with respect to the totality $\psi, t_{i, j}$. Currently problems of high dimension with such constraints can not be numerically solved.

\section{UNEXPECTED TRAFFIC GROWTH}

An unexpected growth of traf $c$ was discussed in [4] separately for OD pairs and for nodes. First we consider OD pairs. Let $T$ be a traf $c$ matrix and $(i, j)$ be an OD pair. Assume that the traf c between $i$ and $j$ increases by $U$. Then it is suggested in [4] to consider a new traf c matrix $T^{\prime}(U) \equiv T^{\prime}(i, j)(U)$ with

$$
t_{i, j}^{\prime}=(1+U) t_{i, j}, t_{j, i}^{\prime}=t_{i, j}^{\prime}
$$

and

$$
t_{i^{\prime}, j^{\prime}}^{\prime}=r_{i, j} t_{i^{\prime}, j^{\prime}},\left(i^{\prime}, j^{\prime}\right) \neq(i, j),\left(i^{\prime}, j^{\prime}\right) \neq(j, i) .
$$

Here $r_{i, j}$ is the coef cient which provides the equality

$$
\sum_{p, q \in V,} t_{p, q}^{\prime}=\sum_{p, q \in V} t_{p, q}
$$

Thus $T^{\prime}$ describes a shift in load without growth. A family of matrix $T^{\prime}(i, j)$ corresponding to each OD pair $(i, j)$ is considered in [4]. In other words it is assumed that an unexpected growth of traf c can occur only for one pair of $\mathrm{OD}$, and this pair is unknown. The growth factor $\psi_{*}\left(T^{\prime}(i, j)\right)$ then calculated for each matrix $T^{\prime}(i, j)$ and the number $\Psi_{*}(T, U)=\min _{(i, j) \in V} \psi_{*}\left(T^{\prime}(i, j)\right.$ is considered as a parameter that characterizes an unexpected traf c growth $U$ corresponding to the matrix $T$. If a collection $\mathcal{T}$ of traf $\mathrm{c}$ matrices $T$ is given, then we need to apply the described procedure for each matrix $T$. Then we get a new collection of traf c matrices, which consists of all matrices $T^{\prime}(i, j)$ for all $T \in \mathcal{T}$ and all $i, j \in V, i \neq j$. The number $\min \left\{\Psi_{*}(T, U): T \in \mathcal{T}\right\}$ characterizes an unexpected traf c growth $U$ corresponding to collection $\mathcal{T}$.

The similar approach was suggested in [4] for the case when single nodes become active. For a particular node $j$ we consider a matrix $\tilde{T} \equiv T(j)$ with

$$
\tilde{t}_{i j}=\tilde{t}_{j i}=(1+U) t_{i j}, i \neq j
$$

and

$$
\tilde{t}_{i j^{\prime}}=r_{j} t_{i j^{\prime}}, j^{\prime} \neq j, i \neq j^{\prime},
$$

where $r_{j}$ is the coef cient which provides the equality

$$
\sum_{p, q \in V} \tilde{t}_{p q}=\sum_{p, q \in V} t_{p q}
$$

The described approach is very interesting and can be used for comparison of different topologies. However, this approach is based on strong enough hypotheses. We only comment on the case of OD pairs. The construction of matrices $T^{\prime}(i, j)$ is based on the assumption that the uniform redistribution of the amount $2 U t_{i, j}$ is carried out between all OD $\left(i^{\prime}, j^{\prime}\right)$ with $\left(i^{\prime}, j^{\prime}\right) \neq(i, j)$ and $\left(i^{\prime}, j^{\prime}\right) \neq(j, i)$. This uniformity does not always hold in real world networks. The assumption that an unexpected growth can occur only for one pair of OD is also not valid in many cases. The following situation should be also taken into account. Assume that unexpected growth occurs for a pair $(i, j)$ at the end of the rst year. This leads to a change of the collection $\mathcal{T}$ of traf $c$ matrices. So we have a different collection $\mathcal{T}^{\prime}$ of traf $\mathrm{c}$ matrices in the second year and cannot use the results obtained for the collection $\mathcal{T}$. This means that we need to recalculate the lifetime measure in the second year. However, the proposed lifetime measure is used for the evaluation of topology design so we should not recalculate it each year. It is therefore important to consider a modi cation to the construction from [4] that is not based on the two mentioned assumptions.

We suggest the consideration of the additive approach rather than the multiplicative approach for the de nition of the lifetime measure.

Let $U$ be a positive number that indicates the total amount of an unexpected traf $c$ growth for the required period of time. Consider the system of linear inequalities

$$
\sum_{p \in P^{*}(i, j ; G)} x_{p} \geq U+t_{i, j}, \quad i, j \in V, i \neq j
$$




$$
\begin{gathered}
\sum_{p \in P_{k, l}^{*}(G)} x_{p} \leq c_{k, l}, \quad(k, l) \in E \\
x_{p} \geq 0, \quad p \in P^{*}(G) .
\end{gathered}
$$

Composing (4) we suppose that unexpected traf $c$ growth $U$ can happen in many arcs and nodes simultaneously. This situation is more realistic than that suggested in [4], where only a single node (or a single ark) becomes more active. Indeed, the increase of activity in a node can lead to the increase of activity in many different nodes. One of the main reasons for unexpected growth is the internet. A server farm, which provides a popular service can suddenly appear and then the load to the corresponding part of network increases. However, the same reasons that lead to appearance of this farm will also lead to appearance of different farms in different parts of network in different years of the period of time $\tau$ under consideration. So it is important to take into account many nodes simultaneously. Note that $U$ is an upper bound for the total unexpected increase of load for the time period $\tau$ for each OD pair $(i, j)$.

The suggested approach is pessimistic, because we assume the worst thing that can happen (unexpected increase in all nodes simultaneously). We are forced to take into account arbitrary unexpected traf $c$ growth if we do not have any forecasts. Usually some forecasts are known. They can narrow the area of unexpected traf $c$ growth. We can either exclude unexpected growth for some links or consider them with coef cients, less than one. This means that instead of (4) we can consider the following:

$$
\sum_{p \in P^{*}(i, j ; G)} x_{p} \geq \alpha_{i j} U+t_{i, j}, \quad i, j \in V, i \neq j
$$

where $0 \leq \alpha_{i j} \leq 1$. For the sake of simplicity we assume that $\alpha_{i j}=1$ for all $i, j$. Note that we do not need to consider links and nodes separately.

In order to check that the system (4)-(6) is feasible it is enough to solve a linear programming problem with an arbitrary linear objective function $f$ and constraints (4) - (6). The most appropriate objective function in this situation is the total traf c:

$$
f(X)=\sum_{p \in P^{*}(G)} x_{p}
$$

where $X=\left(x_{p}\right)_{p \in P^{*}(G)}$. Thus we shall consider the following linear programming problem $\mathrm{LP}(\mathrm{T}, \mathrm{U})$ :

$$
\text { maximize } \sum_{p \in P^{*}(G)} x_{p}
$$

subject to constraints (4)-(6). The system (4)-(6) is feasible if and only if the maximum in (8) is nite. The value of this maximum is denoted by $\lambda(T, U)$. If the system (4)-(6) is unfeasible then the maximum in (8) (that is, the maximum over the empty set) is equal to $-\infty$, so $\lambda(T, U)=-\infty$. It is easy to check that the $\lambda(T, U)$ is a decreasing and concave function of $U$ for each $T$.
The number $\lambda(T, U)$ indicates the greatest possible total traf c that can provide (4) and (5) for a given matrix $T$. We consider this number as a certain lifetime measure of a topology under consideration for the traf c matrix $T$. Let a collection $\mathcal{T}$ of traf $\mathrm{c}$ matrices $T$ be given. Assume that systems (4)-(6) are feasible for each $T \in \mathcal{T}$, then the unexpected traf $\mathrm{c}$ growth $\lambda(\mathcal{T}, U)$ corresponding to $\mathcal{T}$ can be calculated as the minimum of $\lambda(T, U)$ over the set $\mathcal{T}$. We consider $\lambda(\mathcal{T}, U)$ as a lifetime measure for the given topology, the given collection $\mathcal{T}$ and the given set $P^{*}(G)$ of working paths.

Thus we suggest the use of a simple one-step procedure for the de nition of lifetime measure instead of consecutive two-step procedure from [4].

\section{EXTENSIONS OF THE SET OF WORKING PATHS}

Let $U$ be a number such that the problem $\mathrm{LP}\left(T, \mathrm{U}^{\prime}\right)$ is feasible for $U^{\prime}<U$ and $\mathrm{LP}\left(\mathrm{T}, \mathrm{U}^{\prime}\right)$ is unfeasible for $U^{\prime}>$ $U$. Assume that the problem LP(T,U) is feasible and let $\left(\left(x_{p}\right)_{p \in P^{*}(G)}\right)$ be its solution. If all the inequalities (4) hold in the strong sense then there exists $U^{\prime}>U$ such that $\mathrm{LP}\left(\mathrm{T}, \mathrm{U}^{\prime}\right)$ is also feasible, which contradicts the de nition of $U$. Hence, (4) holds as the equality for at least one link. Links $(k, l) \in E$, where the equality holds, indicate the bottleneck that does not permit an unexpected load greater than $U$.

If the load distribution that occurs due to unexpected load growth, exceeds the network possibilities then new facilities should be installed. In order to support the traf $\mathrm{c}$ for the time that is needed for installation of these facilities, the set $P^{*}(G)$ of working paths needs to be extended. We can attract new paths that do not contain links $(k, l)$, where the inequality in (2) holds as the equality. It is easy to nd examples, where even a few such paths allow us to signi cantly increase the capacity to handle an unexpected load.

Clearly it is bene cial to use only fairly short paths in the telecommunication networks. Usually the shortest paths or rst $k$ shortest paths with $k>1$ are considered. It is more convenient to introduce a special notion of the order of a path and then consider paths only with small enough orders. Using this notion we can compare paths for different OD and say for example, that it is bene cial to include new working paths only for OD pairs $\left(i_{1}, j_{1}\right),\left(i_{2}, j_{2}\right),\left(i_{3}, j_{3}\right)$ and consider rst $k$ shortest paths with $k=1$ ( $k=2, k=3$, respectively) for $\left(i_{1}, j_{1}\right)\left(\left(i_{2}, j_{2}\right),\left(i_{3}, j_{3}\right)\right.$, respectively).

\section{ORDER OF A PATH}

Different notions of the order of a path can be introduced for estimating its quality. We consider three different notions of the order.

Let $v, v^{\prime} \in V, v \neq v^{\prime}$. The length of the shortest path with the end -nodes $v$ and $v^{\prime}$ is denoted by $\lambda\left(v, v^{\prime}\right)$. Consider now a path $p=\left(v, \ldots, v_{i} \ldots, v^{\prime}\right)$ with the same end-nodes and the length $\lambda(p)$. The number $\sigma_{1}(p)=\frac{\lambda(p)}{\lambda\left(v, v^{\prime}\right)}$ indicates how many super uous links are contained in the path $p$. Note that $\sigma_{1}(p) \geq 1$. If $\sigma_{1}(p)$ is a large number then the path $p$ contains 
many super uous links. We can consider $\sigma_{1}(p)$ as the order of the path $p$.

The order $\sigma_{1}(p)$ is not suitable for the description of a situation, where only a certain part of the path contains super uous links.

Example 6.1: Consider a path $p=\left(v_{1}, \ldots, v_{t}, v_{t+1}, \ldots\right.$, $\left.v_{t+s}\right)$, where $\left(v_{1}, \ldots, v_{t}\right)$ is the shortest path between $v_{1}$ and $v_{t}$ and there is link $l_{v_{t}, v_{t+s}}$, such that the shortest path between $v_{1}$ and $v_{t+s}$ is $\left(v_{1}, \ldots, v_{t}, v_{t+s}\right)$. Then $\lambda\left(v_{1}, v_{t+s}\right)=t+1$ and $\lambda(p)=t+s$ so $\sigma_{1}(p)=\frac{t+s}{t+1}$. Assume that $s$ is xed. If $t$ is a large enough number then $\sigma_{1}(p)$ can be closed to 1 , so accordingly to the factor $\sigma_{1}$, the whole path $p$ is good enough. However the sub-path $\left(v_{t}, \ldots, v_{t+s}\right)$ contains many super uous links.

In order to re ect the in uence of sub-paths to the quality of a path we can use the following construction. Consider a path $p=\left(v_{0}, v_{1}, \ldots, v_{t-1}, v_{t}\right)$ and all sub-paths $p_{i, j}=\left(v_{i}, \ldots, v_{j}\right)$ of this path. Let

$$
\sigma_{2}(p)=\max _{i=0, \ldots, k-1, i<j \leq t} \sigma_{1}\left(p_{i, j}\right)
$$

The number $\sigma_{2}(p)$ can be considered as another order of a path $p$. This order indicates the quality of the worst sub-path of this path. If this number is large then the path contains a sub-path with relatively large amount of super uous links, In contrast with $\sigma_{1}(p)$, the number $\sigma_{2}(p)$ does not re ect the quality of the whole path. We can consider one of the numbers $\sigma_{1}(p), \sigma_{2}(p)$ or both of them for estimation of the quality of a path. Sometimes it is convenient to consider a combination of orders $\sigma_{1}(p)$ and $\sigma_{2}(p)$. A number

$$
\sigma(p)=\frac{1}{2}\left(\sigma_{1}(p)+\sigma_{2}(p)\right)
$$

also can be considered as an order of the path $p$, which re ects the in uence of a path itself and its sub-paths. We use this characteristic as the order. The order of a path is a convenient tool for comparison of different paths of the same length.

In the same manner we can de ne the order of a path in terms of cost. It is easy to nd examples where $k$-shortest paths between different OD $(i, j)$ with the same $k$ have different orders. In order to increase the growth factor, we can use some additional working paths with the small order $\sigma$.

\section{USING FORECAST OF GROWTH OF POPULATION}

In order to have a more precise lifetime measure we need to use forecasts of growth of population and migrations ows. Usually such forecasts are known. We can suppose that traf c between two nodes is proportional to the population of these nodes with a certain coef cient of proportionality $\gamma$ which depends on different issues, in particular, on the rate of migration. Assume the a forecast suggests that the set of nodes $V$ can be presented as the union of two disjoint sets $V^{\prime}$ and $V^{\prime \prime}: V=V^{\prime} \cup V^{\prime \prime}$. The population of nodes $v^{\prime} \in V^{\prime}$ will grow and the population of nodes $v^{\prime \prime} \in V^{\prime \prime}$ will remain the same. Let $u$ be the rate of growth of population at cities $i \in V^{\prime}$ (We assume for the sake of simplicity that both $u$ and the coef cient of proportionality $\gamma$ do not depend on a node $i \in V^{\prime}$.) Let $\mathcal{T}$ be the set of current traf $c$ matrices and $T=\left(t_{i j}\right) \in \mathcal{T}$. Then a new traf c matrix $S_{T}(u)$ has entries $s_{i j}$ where

$$
\begin{gathered}
s_{i j}=\gamma^{2}(1+u)^{2} t_{i j}, \quad i, j \in V^{\prime} \\
s_{i j}=\gamma(1+u) t_{i j}, \quad i \in V^{\prime}, j \in V^{\prime \prime} \\
s_{i j}=t_{i j}, \quad i, j \in V^{\prime \prime}, j \in V^{\prime \prime} .
\end{gathered}
$$

The set of matrices $\mathcal{S}=\left\{S_{T}(u): T \in \mathcal{T}\right\}$ should be considered instead of $\mathcal{T}$ for the calculation of the lifetime measure in the situation under consideration.

\section{USING SUBGRAPHS}

Different parts of the network have a different in uence on the lifetime measure. Thus we need to consider the developed approaches not only for a given graph $G$ but also for some its subgraphs $G^{\prime}$. In order to do this we need to determine the restriction of the traf c matrices $T$ to a subgraph $G^{\prime}$. The following de nition is useful. A node $i \in V^{\prime}$ is called an outer node for $G^{\prime}$ if there are links $(k, i) \in E$ with $k \notin V^{\prime}$ and $(i, j)$ with $j \in V^{\prime}$.

Let $G^{\prime}=\left\langle V^{\prime}, E^{\prime}\right\rangle$ be a subgraph of $G$. Denote by $V^{\prime \prime}$ the complement to $V^{\prime}: V^{\prime \prime}=V \backslash V^{\prime}$. We now classify all paths from $P^{*}(G)$ with respect to $G^{\prime}$, namely we consider three classes of paths.

1) Paths $p$, which do not go through $G^{\prime}$. This means that $p$ does not contain links from $E^{\prime}$. Note that some nodes of $p$ can belong to $V^{\prime}$, in such a case these are outer nodes of $V^{\prime}$. 2) Paths $p$, which go through $G^{\prime}$ once. We say that a path $p$ goes through $G^{\prime}$ once if $p$ has the form

$$
p=(v, \ldots, i, \ldots, j, \ldots, u)
$$

where $i, j \in V^{\prime}, i \neq j$ and the path $p^{\prime}=(i, \ldots, j)$ is located in $G^{\prime}$ (the latter means that $p^{\prime}$ consists of links, which belong to $E^{\prime}$ ); It is possible that $v=i$ and/or $j=u$. If $v \neq i$, then $i$ is an outer node and the path $(m, \ldots, i)$ does not go through $G^{\prime}$; if $j \neq u$ then $j$ is an outer node and $(j, \ldots, u)$ does not go through $G^{\prime}$. If $v=i$ and $j=u$ then $p$ is a path with the end-nodes $i$ and $v$, which is located in $G^{\prime}$. Thus an arbitrary path, which is located in $G$, is going through $G^{\prime}$ once.

3) Paths $p$, which go through $G^{\prime}$ more than once. This means that $p$ contains more than one piece of the form $\left(i_{\alpha}, \ldots, j_{\alpha}\right)$ with $i_{\alpha} \neq j_{\alpha}$, which is located in $G^{\prime}$.

The analysis of paths, which go through $G^{\prime}$ more than once is dif cult. The simplest way to handle these paths is to divide them into parts which go through $G^{\prime}$ once and examine these parts separately. Thus we can assume that the set $P^{*}(G)$ contains only paths that either do not go through $G^{\prime}$ or go through $G^{\prime}$ once. We use the following notation:

$P_{G^{\prime}}^{*}(G)$ is the set of all working paths $p \in P^{*}(G)$ going through $G^{\prime}$ once.

$P_{G^{\prime}}^{*}(i, j ; G)$ is the set of all working paths of the form (9) with xed $i, j \in V^{\prime}$. 
If nodes $i, j \in V^{\prime}$ are not outer nodes of $G^{\prime}$ then $P_{G^{\prime}}\left(i, j ; G^{\prime}\right)$ coincides with the set $P\left(i, j ; G^{\prime}\right)$ of all paths of $G^{\prime}$ with the end-nodes $i$ and $j$.

Let $U$ be a size of an unexpected growth in traf c and let $\left(\widetilde{x}_{p}\right)_{p \in P^{*}}$ be the set of paths such that $\left(\left(\bar{x}_{p}\right)_{p \in P^{*}(G)}\right.$ is a solution of problem LP(T,U). Let $G^{\prime}=\left\langle V^{\prime}, E^{\prime}\right\rangle$ be a subgraph of the graph $G$ and let $i, j \in V^{\prime}, i \neq j$. Consider a total ow $y_{i j}$, which should be transmitted from the node $i$ to the node $j$ through paths from $P^{*}\left(G^{\prime}\right)$, which go through $G^{\prime}$ once:

$$
y_{i, j}=\sum_{\left.p \in P_{G^{\prime}}^{*}(i, j ; G)\right)} \bar{x}_{p} .
$$

We also put $y_{i i}=0$. Consider the matrix $Y=\left(y_{i j}\right)_{i, j \in V}$. Clearly $Y$ is symmetric, $y_{i j} \geq 0$ and $y_{i i}=0$ for all $i$ so we can consider $Y$ as a traf $\mathrm{c}$ matrix for the undirected traf $\mathrm{c}$ corresponding to the subgraph $G^{\prime}$.

If $i$ and $j$ are not outer nodes of $V^{\prime}$ then $P_{G^{\prime}}^{*}(i, j ; G)$ coincides with $P^{*}\left(i, j ; G^{\prime}\right)$, hence the boundary conditions $y_{i, j}$ coincides with traf c demands $t_{i j}$. If $i$ is an outer node and $j$ is not an outer node, then $y_{i, j}$ is the total ow on the paths $p=(v, \ldots, i, \ldots, j)$, which go from either endnodes $v$ outside $G^{\prime}$ or $i$ through $G^{\prime}$ once to $j$. If both $i$ and $j$ are outer nodes, then $y_{i, j}$ is the total ow, on the paths $p=(v, \ldots, i, \ldots, j, \ldots, u)$, which go once through $G^{\prime}$. (Here either $v \notin V^{\prime}$ or $v=i$ and either $u \notin V^{\prime}$ or $u=j$.)

The matrix $Y$ depends on a traf c matrix $T$, subgraph $G^{\prime}$ and a ow $\left(\bar{x}_{p}\right)$. We can consider a procedure suggested in Section IV and calculate the number $\lambda(Y, U)$ for the given matrix $Y$. Since $V^{\prime}$ is smaller than $V$, the calculation of $\lambda(Y, U)$ is simpler than the calculation of $\lambda(T, U)$.

\section{CONCLUSIONS}

We have discussed some issues related to lifetime measures for telecommunication networks. Such measures are important for comparing networks. A lifetime measure should take into account not only the expected growth in demand but also an unexpected traf $\mathrm{c}$ growth.
We demonstrated some advantages and disadvantages of the topology lifetime measure that was introduced in the pioneering paper [4]. In particular, we showed that this measure is based on strong enough hypotheses. We introduced a more realistic lifetime measure and discussed its properties.

Lifetime measures depend not only on the topology of the network but also on the set of working paths. Sometimes this set should be extended (for example, we need to extend this set in order to support the traf $\mathrm{c}$ for the time that is needed for installations of new facilities). It is convenient to use special measure for comparing paths in order to choose new working paths. We suggest a possible measure (the order of a path) for comparing paths and discuss its properties.

We show that forecasts of growth of population and migration ows can be used in the de nition of lifetime measures.

Different parts of the network have a different in uence on the lifetime measure, so it is important to examine the restriction of a given traf $\mathrm{c}$ to some parts of the network. We suggest an approach to such an examination.

\section{REFERENCES}

[1] Pickavet M., Demeester P., îulti-Period Planning of Survivable WDM Networksî, European Transaction on Telecommunication, Special Issue on WDM Networks, Vol.11, pp.7-16, 2000.

[2] Rajogopalan S., Singh M., Morton T., îCapacity Expansion and Replacement in Growning Markets with Uncertain Technological Breakthroughsî, Management Science, Vol. 44, pp12-30, 1998.

[3] Gryseels M., Sorbello L., Demeester P., îPlanning in Uncertain and Dynamic Environmentsî, Proc. 9th International Netwotk Planning Symposium, Toronto, 2000.

[4] Maxemchuk, N., Ouveysi, I. and Zukerman, M., îA Measure of Network Lifetime,î Proceedings of the 9th IEEE International Workshop on Computer-Aided Modelling, Analysis and Design of Communication Links and Networks (CAMAD 2002), May 2002.

[5] Maxemchuk, N., Ouveysi, I. and Zukerman, M., iA Measure of Network Lifetime, netlab.caltech.edu/CAMAD02/08Nick-Maxemchunk.pdf

[6] Dzalilov Z., Ouveysi I. and Rubinov A., îDynamic Recon guration of Telecommunication Networksî. Proceeding of Industrial Optimisation Symposium, Curtin University of Technology, Perth, 2003 